\title{
A novel diagnostic signature based on three circulating exosomal mircoRNAs for chronic obstructive pulmonary disease
}

\author{
YAHUI SHEN ${ }^{1,2}$, LINA WANG $^{1}$, YUNHUI WU ${ }^{1}$, YINGWEI OU ${ }^{1}$, HUIYU LU $^{2}$ and XIN YAO ${ }^{1}$ \\ ${ }^{1}$ Department of Respiratory and Critical Care Medicine, The First Affiliated Hospital of Nanjing Medical University, \\ Nanjing, Jiangsu 210029; ${ }^{2}$ Department of Respiratory and Critical Care Medicine, Taizhou Clinical Medical School of \\ Nanjing Medical University, Taizhou, Jiangsu 225300, P.R. China
}

Received October 31, 2020; Accepted March 18, 2021

DOI: $10.3892 /$ etm.2021.10149

\begin{abstract}
Exosomal microRNAs (exo-miRNAs or miRs) have demonstrated diagnostic value in various diseases. However, their diagnostic value in chronic obstructive pulmonary disease (COPD) has yet to be fully established. The purpose of the present study was to screen differentially expressed exo-miRNAs in the plasma of patients with COPD and healthy individuals and to evaluate their potential diagnostic value in COPD. Differentially expressed exo-miRNAs in the plasma of patients with COPD and controls were identified using high-throughput sequencing and confirmed using reverse transcription-quantitative PCR (RT-qPCR). Bioinformatics analysis was then performed to predict the function of the selected exo-miRNAs and their target genes in COPD. After a network model was constructed, linear regression analysis was performed to determine the association between exo-miRNA expression and the clinical characteristics of subjects in a validated cohort (46 COPD cases; 34 matched healthy controls). Receiver operating characteristic curve was subsequently plotted to test the diagnostic value of the candidate biomarkers. The top 20 significantly aberrantly expressed COPD-associated exo-miRNAs were verified using RT-qPCR.
\end{abstract}

Correspondence to: Professor Xin Yao, Department of Respiratory and Critical Care Medicine, The First Affiliated Hospital of Nanjing Medical University, 300 Guangzhou Road, Nanjing, Jiangsu 210029, P.R. China

E-mail: groupyaoxin@163.com

Abbreviations: COPD, chronic obstructive pulmonary disease; miRNAs, microRNAs; Exo-miRNA, exosomal microRNA; TSG101, tumor susceptibility gene 101; RT-qPCR, reverse transcription-quantitative PCR; KEGG, Kyoto Encyclopedia of Genes and Genomes; GO, Gene Ontology; ROC, receiver operating characteristic; AUC, area under the curve; FEV1, forced expiratory volume in the 1st second; FVC, forced vital capacity

Key words: exosome, microRNA, chronic obstructive pulmonary disease, biomarkers, diagnosis
Of these, nine were then selected for subsequent analysis, five of which were found to be upregulated (miR-23a, miR-1, miR-574, miR-152 and miR-221) and four of which were downregulated (miR-3158, miR-7706, miR-685 and miR-144). The results of Gene Ontology and KEGG pathway analysis revealed that these miRNAs were mainly involved in certain biological functions, such as metabolic processes, such as galactose metabolism and signaling pathways (PI3K-AKT) associated with COPD. The expression levels of three exo-miRNAs (miR-23a, miR-221 and miR-574) were found to be negatively associated with the forced expiratory volume in the 1st second/forced vital capacity. Furthermore, the area under the curve values of the three exo-miRNAs (miR-23a, miR-221 and miR-574) for COPD diagnosis were 0.776 [95\% confidence interval (CI), 0.669-0.882], 0.688 (95\% CI, 0.563-0.812) and 0.842 (95\% CI, 0.752-0.931), respectively. In conclusion, the three circulating exosomal miRNAs (miR-23a, miR-221 and miR-574) may serve as novel circulating biomarkers for the diagnosis of COPD. These results may also enhance our understanding and provide novel potential treatment options for patients with COPD.

\section{Introduction}

Chronic obstructive pulmonary disease (COPD) is a common respiratory disease with a high rate of morbidity and mortality (1-3). As the rates of COPD incidence have increased, so too has its social and economic burden. It is estimated that by 2020, COPD will rank fifth in the list of diseases that most impacts the world's economy (4). The pathological characteristics of COPD include chronic inflammation of the lung parenchyma and the surrounding airways, emphysema and small airway stenosis or remodeling (5-7). A number of processes, including oxidative stress, inflammation and mitochondrial dysfunction have all been reported to serve mechanistical roles (8-11). However, additional research is required to determine the molecular mechanisms underlying COPD.

MicroRNAs (miRNA or miRs) are a group of non-coding RNAs that consist of 18-25 nucleotides and serve to increase mRNA degradation or repress target-specific mRNA 
translation (12). miRNAs can modulate almost all biological processes, including proliferation, differentiation, inflammatory response, autophagy, tissue remodeling, immune regulation, angiogenesis, aging and tumorigenesis (13-15). Previous reports have confirmed that miRNAs can also serve an important role in the pathology of COPD (16-18). Therefore, regulating miRNA expression may be a potential therapeutic strategy for COPD.

Exosomes are nano-sized membrane-bound vesicles that are released into the extracellular environment by almost all cell types (19-21). Once stimulated by inflammation, hypoxia, oxidative stress and immune activation, macromolecules contained inside exosomes, such as DNA and RNA, are transformed and exuded to affect the biology of receptor cells (22-24). In addition, exosomes can be successfully isolated from different types of body fluids, including sputum, plasma and bronchoalveolar lavage (25-27). Exosomes contain non-coding RNA, protein, lipid and other substances, which change with the state of diseases including COPD (28). This implies that there may be an association between exosomes and COPD pathogenesis (29).

Unfortunately, sensitive biomarkers that can potentially be used for the prognosis of COPD remain lacking. Previous studies have demonstrated that the levels of miR-29c and miR-126 in the peripheral blood are potential biomarkers for the diagnosis of COPD $(30,31)$. However, the miRNA in the peripheral circulation is susceptible to interference by other components, making circulating miRNA results less consistent. Exosomes have a dual-membrane structure that protects them from being degraded by ribonucleases, rendering exosomal miRNAs (exo-miRNAs) ideal for use as circulating biomarkers (32).

Therefore, the present study isolated exosomes from the plasma samples of patients with COPD and healthy individuals. High-throughput sequencing was performed to identify enriched miRNAs in the exosomes of patients with COPD. The exo-miRNAs closely associated with the clinical characteristics of COPD were subsequently screened and their diagnostic values were analyzed.

\section{Materials and methods}

Study subjects. The present study was approved by the Research Ethics Committee of Taizhou Clinical Medical School of Nanjing Medical University (approval no. KY201904701; Taizhou, China) and conducted according to the Declaration of Helsinki. All the selected patients voluntary participated and provided their written informed consent.

Between January 2019 and August 2020, a total of 46 patients with COPD were selected for the current study at Taizhou Clinical Medical School of Nanjing Medical University (Taizhou, China). At the same time, 34 healthy non-smoking individuals (range, 45-85 years) were recruited as the controls at Taizhou Clinical Medical School of Nanjing Medical University (Taizhou, China). To reduce heterogeneity, the age and sex of the healthy controls were matched to the patients with COPD. The average age of the patients with COPD was 62 years (age range, $45-85$ years) with moderate to severe pulmonary dysfunction [the post bronchodilator ratio of the forced expiratory volume in the 1st second (FEV1) to the forced vital capacity (FVC) value, $<0.70$; FEV1\% predicted value, $<80 \%$ ], were included in the present study. COPD diagnosis and severity classification were established by the same respiratory physician HL, according to the GOLD criteria (33). Patients with COPD were either current or ex-smokers (lifetime smoking exposure, smoking index $\geq 10$ pack-years; smoking cessation, $<1$ year). Prior to enrollment, patients were included if they had a documented history of at $\geq$ two COPD exacerbations leading to oral or intravenous glucocorticoid therapy or antibiotic therapy, or at $\geq$ one COPD exacerbation leading to hospitalization within the previous year. Patients were excluded if they presented with other diseases, including severe cardiovascular disease, uncontrolled high blood pressure, bleeding, hepatic failure, renal failure, rheumatoid immune disease and malignant tumors.

Study design. The present study was divided into two sections: Discovery and validation. In the discovery stage (stage I), high-throughput sequencing was performed to screen the differentially expressed plasma-derived exo-miRNAs between samples from the group of five patients with COPD and the group with five healthy individuals. The function of differentially expressed miRNAs was evaluated using Gene Ontology (GO) and Kyoto Encyclopedia of Gene and Genomes (KEGG) pathway analysis. Exosomal miRNAs exhibiting the most significant (according to the multiple of differential expression calculated by $\log _{2} \mathrm{FC}$; $\left.\mathrm{P}<0.05\right)$ differential expression were selected for further validation.

In the validation stage (stage II), the expression of the potential plasma-derived exo-miRNAs in 41 patients with COPD and 29 healthy individuals were analyzed using reverse transcription-quantitative PCR (RT-qPCR). Receiver operating characteristic (ROC) curve-based risk assessment analysis was performed to evaluate the sensitivity and specificity of using exo-miRNAs to diagnose COPD.

Plasma sample collection. After overnight fasting, $20 \mathrm{ml}$ whole blood was drawn from each participant through the antecubital vein and injected into an EDTA-coated vacutainer tube (lavender top EDTA tubes; BD Biosciences). The tubes were incubated at $4^{\circ} \mathrm{C}$ for $3-4 \mathrm{~h}$ and centrifuged at $4,000 \mathrm{x}$ g at $4^{\circ} \mathrm{C}$ for $5 \mathrm{~min}$. The supernatants containing plasma were collected and stored in a $2-\mathrm{ml}$ falcon tube at $-80^{\circ} \mathrm{C}$ for further analysis.

Exosome isolation and characterization. Plasma-derived exosomes were isolated using an ExoQuick commercial kit Ribo $^{\mathrm{TM}}$ Exosome Isolation Reagent (Guangzhou RiboBio Co., Ltd.) according to the manufacturer's protocol. Transmission electron microscopy (TEM) was performed to observe the size and morphology of exosomes as described previously $(34,35)$. Exosomes were placed on a copper mesh surface, fixed with $3 \%$ glutaraldehyde at room temperature for $5 \mathrm{~min}$ and stained with $4 \%$ uranium acetate at room temperature for $1 \mathrm{~min}$. Finally, the copper mesh was transferred to $1 \%$ methylcellulose and suspended at room temperature for $3 \mathrm{~min}$. Excess liquid at the edge of the copper mesh was dried using filter paper for $\geq 30 \mathrm{~min}$. Stained samples were observed using TEM (magnification, x80,000) (Hitachi-7650; Hitachi, Ltd.) and imaged for preservation. Nanoparticle tracking analysis (NTA) was used to evaluate the size and concentration of 
plasma exosomes. Brownian particles were tracked, following which their hydrodynamic diameters and concentrations were calculated using the Stockes-Einstein equation (36). NTA assay was performed using the ZetaView PMX 110 (Particle Metrix GmbH). The data obtained with the ZetaView instrument were analysed using the corresponding software ZetaView v.8.02.28 (Particle Metrix GmbH).

Western blotting. Western blotting was used to detect the specific marker proteins (CD9, CD63, TSG101) on the surface of plasma exosomes. Exosome samples were lysed using RIPA lysis buffer (cat. no. 20-188; Sigma-Aldrich, Merck KGaA) on ice for $30 \mathrm{~min}$. A bicinchoninic acid (BCA) Protein Assay kit (cat. no. 23225; Pierce; Thermo Fisher Scientific, Inc.) was used to detect protein concentrations, and a $12 \%$ sodium dodecyl sulfate-polyacrylamide gel was used for total protein $(30 \mu \mathrm{g} /$ lane) separation. The proteins in the gel were transferred to a $0.45-\mu \mathrm{M}$ pore size PVDF membrane (cat. no. IPVH00010; EMD Millipore) via wet electrophoretic transfer. The membranes were blocked with 5\% skimmed milk powder for $1 \mathrm{~h}$ at room temperature and incubated overnight at $4^{\circ} \mathrm{C}$ with anti-CD9 (cat. no. ab92726; Abcam), anti-CD63 (cat. no. ab216130), anti-TSG101 (cat. no. ab125011) antibodies, all diluted to $1: 1,000$ in TBS-1\% Tween-20. Subsequently, the membranes were incubated with a horseradish peroxidase-conjugated goat anti rabbit (1:3,000; cat. no. A0208; Beyotime Institute of Biotechnology) or goat anti mouse IgG secondary antibodies (1:3,000; cat. no. A0216; Beyotime Institute of Biotechnology) for $1 \mathrm{~h}$ at room temperature. The membranes were visualized using LumiBest enhanced chemiluminescence (cat. no. SB-WB011; Shanghai Shenger Biotechnology Co., Ltd.).

Exosomal miRNA sequencing. A total of $4 \mathrm{ml}$ plasma was mixed with Ribo ${ }^{\mathrm{TM}}$ Exosome Isolation Reagent (Guangzhou RiboBio Co., Ltd.), after which exosomes were isolated. Exosomal RNA was extracted using the HiPure Plasma miRNA kit (cat. no.R314; Guangzhou Guangzhou Meiji Biological Technology Co., Ltd.). The quantity and integrity of exosomal RNA was assessed using the Qubit ${ }^{\circledR} 2.0$ (Thermo Fisher Scientific, Inc.) and Agilent 2200 TapeStation (Agilent Technologies, Inc.), respectively. For each sample, $50 \mathrm{ng}$ exosomal RNA was used to prepare small RNA libraries using the NEBNext ${ }^{\circledR}$ Multiplex Small RNA Library Prep Set for Illumina (cat. no. E7580; New England BioLabs ${ }^{\circledR}$ Inc.). Libraries were sequenced using HiSeq 2500 (Illumina, Inc.) with single-end 50 bp at Guangzhou RiboBio Co., Ltd.. The final loading concentration of each sample was above $2.5 \mathrm{nmol} / \mathrm{l}$. The raw reads were processed by filtering out containing adapter, poly 'N', low quality, smaller than $17 \mathrm{nt}$ reads by FastQC v.0.11.9 (https://directory.fsf.org/wiki/FastQC) to get clean reads. Mapping reads were obtained by mapping clean reads to reference genome of by BWA v.0.7.12 (37). The differential expression between two sets of samples was calculated using the edgeR algorithm (38): $\log _{2}$ (fold change) $\geq 1$ and $\mathrm{P}<0.05$. R software v.3.1.3 (www.r-project.org) was used for data analysis.

$R T-q P C R$ validation. As previously described (32), plasma samples were thawed and centrifuged at $1,000 \mathrm{x} \mathrm{g}$ for $5 \mathrm{~min}$ at room temperature to form granules. The absorbance of oxyhemoglobin was then determined via spectrophotometry at $414 \mathrm{~nm}$. Once hemolysis occurred, the specimen was discarded. Total RNA was extracted from plasma exosomes using TRIzol ${ }^{\circledR}$ reagent (Invitrogen; Thermo Fisher Scientific, Inc.). The primers (One RT primer and a pair of qPCR primers for each set) specific for miRNAs were designed by RiboBio (Guangzhou RiboBio Co., Ltd.). The primer sequences were patented. cDNA was synthesized from 500 ng RNA using a reverse transcriptase kit (cat. no. R10031.8; Guangzhou RiboBio Co., Ltd.) with the following temperature protocol: $42^{\circ} \mathrm{C}$ for $60 \mathrm{~min}$, and followed by $70^{\circ} \mathrm{C}$ for $10 \mathrm{~min}$. qPCR was carried out in accordance with the protocols of the Bulge-Loop ${ }^{\mathrm{TM}}$ miRNA qRT-PCR Starter kit (cat. no. c10211-1; Guangzhou RiboBio Co., Ltd.) and ABI Prism 7900HT (Applied Biosystems; Thermo Fisher Scientific, Inc.). The following thermocycling conditions were used: Initial denaturation at $95^{\circ} \mathrm{C}$ for $20 \mathrm{sec}$, followed by 40 cycles of $95^{\circ} \mathrm{C}$ for $10 \mathrm{sec}, 60^{\circ} \mathrm{C}$ for $20 \mathrm{sec}$ and $70^{\circ} \mathrm{C}$ for $10 \mathrm{sec}$. U6 small nuclear RNA and cel-miR-39 were used as endogenous (39) and exogenous controls, respectively. The expression of exo-miRNAs was calculated using the $2^{-\Delta \Delta \mathrm{Cq}}$ method (40).

GO and KEGG pathway analysis. TargetScan (version 7.2, https://www.targetscan.org/), miRDB (version 5.0, http://www. targetscan.org/), miRTarBase (version 7.0, http://starbase.sysu. edu.cn/) and miRWalk (version 7.0, http://mirwalk.umm. uni-heidelberg.de/) databases were used to predict the target genes of selected miRNAs. Those simultaneously predicted by $\geq$ two of the tools aforementioned were selected as candidate target genes. KOBAS release number 3.0 (http://kobas.cbi. pku.edu.cn/) was used for further GO and KEGG pathway analyses (41). The significance of GO and KEGG pathway analysis was then determined using Fisher's exact and $\chi^{2}$ tests. The subsequent P-value was corrected using the false discovery rate (FDR). GO and KEGG terms with an adjusted $\mathrm{P}$-value $<0.05$ and an FDR $<0.05$ were selected and considered to be enriched.

miRNA-mRNA network construction. An exosome miRNA-mRNA network was constructed. Pearson's correlation of each gene pair was calculated and pairs with significant correlations were selected for network creation. Exo-miRNAs and mRNAs with Pearson correlation coefficients $\geq 0.99$ were selected to construct the network using Cytoscape bioinformatics software v.3.8.0 (https://cytoscape.org/) (42). During network analysis, connectivity was defined as the number of connections between nodes. In addition, the degree (the number of target genes targeted by miRNAs) was subsequently calculated, which is the simplest and most important measure used to determine the relative importance of genes in a network (43).

Statistical analysis. Demographic and clinical characteristics were presented as the mean \pm standard deviation. Experiments were repeated at least 3 times. All analyses were performed using SPSS 24.0 software (IBM Corp.) and GraphPad Prism 5 software (GraphPad Software, Inc.). Student's paired t-test and Mann-Whitney unpaired test analysis were used to evaluate statistical differences between patients with COPD and controls. Categorical variables were analyzed using the 
Table I. Characteristics of patients.

\begin{tabular}{lccr}
\hline Parameters & Control & COPD & P-value \\
\hline Number of subjects & 34 & 46 & NA \\
Male/Female & $27 / 7$ & $36 / 10$ & NA \\
Age, years (range) & $61.2 \pm 6.3$ & $62.3 \pm 5.6$ & $>0.05$ \\
Smoking, pack years & N/A & $52.6 \pm 12.5$ & $<0.001$ \\
Smoking/currently smoking & N/A & 46 & NA \\
Predicted FEV1\% & $97.8 \pm 9.1$ & $49.0 \pm 13.7$ & $<0.001$ \\
FEV1/FVC\% & $81.6 \pm 5.3$ & $54.8 \pm 12.6$ & $<0.001$ \\
Inhaled corticosteroids & 0 & $46 / 46$ & $<0.001$
\end{tabular}

FEV1, Forced Expiratory Volume in $1 \mathrm{sec}$; FVC, Forced Vital Capacity; COPD, chronic obstructive pulmonary disease; NA, not applicable.

Table II. Top 10 miRNAs exhibiting the most significant difference in expression levels.

\begin{tabular}{llll}
\multicolumn{4}{l}{ A, Upregulated } \\
\hline Rank & \multicolumn{1}{c}{ miRNAs } & $\log _{2}(\mathrm{FC})$ & P-value \\
\hline 1 & hsa-miR-23a & 3.0915 & 0.0047 \\
2 & hsa-miR-3138 & 2.8479 & 0.0214 \\
3 & hsa-miR-1268a & 2.7234 & 0.0161 \\
4 & hsa-miR-143 & 2.6790 & 0.0041 \\
5 & hsa-miR-6515 & 2.5967 & 0.0084 \\
6 & hsa-miR-1 & 2.2773 & 0.0094 \\
7 & hsa-miR-221 & 2.0883 & 0.0493 \\
8 & hsa-miR-574 & 2.0538 & 0.0271 \\
9 & hsa-miR-335 & 1.7488 & 0.0204 \\
10 & hsa-miR-152 & 1.7204 & 0.0364 \\
\hline
\end{tabular}

B, Downregulated

\begin{tabular}{clcc}
\hline Rank & \multicolumn{1}{c}{ miRNAs } & $\log _{2}(\mathrm{FC})$ & P-value \\
\hline 1 & hsa-miR-6859 & -2.9372 & 0.0303 \\
2 & hsa-miR-3682 & -2.8839 & 0.0365 \\
3 & hsa-miR-3158 & -2.8433 & 0.0311 \\
4 & hsa-miR-147b & -2.7802 & 0.0338 \\
5 & hsa-miR-7706 & -2.7076 & 0.0300 \\
6 & hsa-miR-6850 & -2.4224 & 0.0217 \\
7 & hsa-miR-144 & -2.3069 & 0.0382 \\
8 & hsa-miR-3192 & -2.1125 & 0.0307 \\
9 & hsa-miR-7704 & -2.0504 & 0.0102 \\
10 & hsa-miR-1299 & -1.9180 & 0.0017 \\
\hline
\end{tabular}

miRNAs are arranged in descending order from highest to lowest. miR, microRNA; $\log _{2}$ (FC), $\log _{2}$ (Fold change).

$\chi^{2}$ test. The differential expression of miRNAs was evaluated via the U Mann Whitney test. Furthermore, Spearman's linear regression method, with Bonferroni's correction for multiple comparisons, was used to analyze the correlation between lung function parameters (FEV1/FVC) and the expression of miRNAs. The ROC curve was used to analyze the efficiency of exo-miRNA as a biomarker for COPD diagnosis. The sensitivity and specificity of each exo-miRNA to diagnose COPD were calculated using the area under the ROC curve (AUC) with $95 \%$ confidence intervals (CI). The optimal cut off point was where the 'true positive rate' was high and the 'false positive rate' was low. Youden's index was determined to identify the optimal cut-off point for calculating exact diagnostic indices. $\mathrm{P}<0.05$ was considered to indicate a statistically significant difference.

\section{Results}

Patient clinical characteristics. In the present study, a total of 80 individuals were enrolled, including 46 patients with COPD and 34 healthy controls (Table I). There was no difference in age and sex between the two groups.

Exosome isolation and identification. Extracted exosomes were found to be $50-150 \mathrm{~nm}$ in size and exhibited round or spindle shapes (Fig. 1A). No significant difference was observed in the size or total number of exosomes between patients with COPD and healthy controls (Fig. 1B). Exosome markers TSG101, CD63 and CD9 were also highly expressed in the exosomes isolated from the plasma samples of the two groups (Fig. 1C).

Exosomal miRNAs are dysregulated in patients with COPD. The exosomal miRNA profiles of five patients with COPD and five healthy individuals were assessed (Fig. 2). Compared with those in healthy individuals, 39 upregulated and 20 downregulated exo-miRNAs were identified in patients with COPD. The top 20 miRNAs with the most significant differential expression are presented in Table II. These miRNAs were selected for further functional analysis.

GO and KEGG pathway analysis. GO analysis revealed that the most enriched biological processes were 'single-organism process', 'metabolic process' and 'biological regulation'. The top enriched cellular components were 'intracellular part', 'membrane-bounded organelle', 'cytoplasm' and 'cytoplasmic part'. Finally, the top enriched molecular functions included 
A

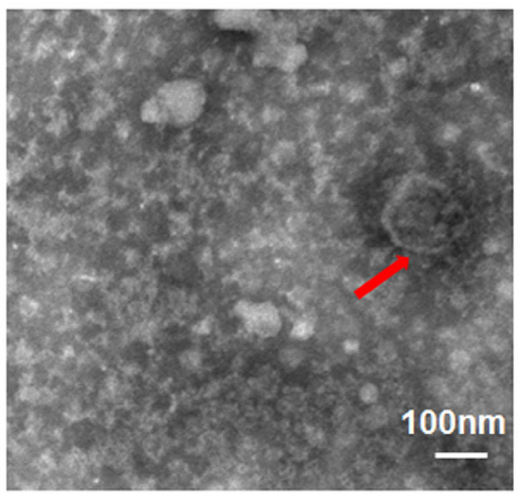

Control

B

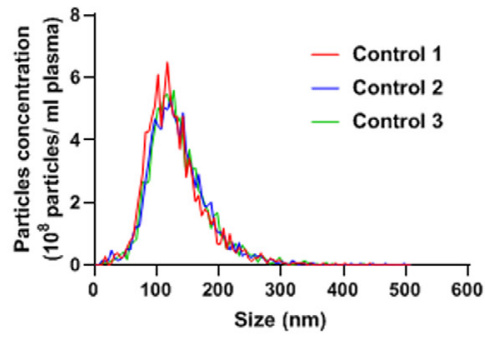

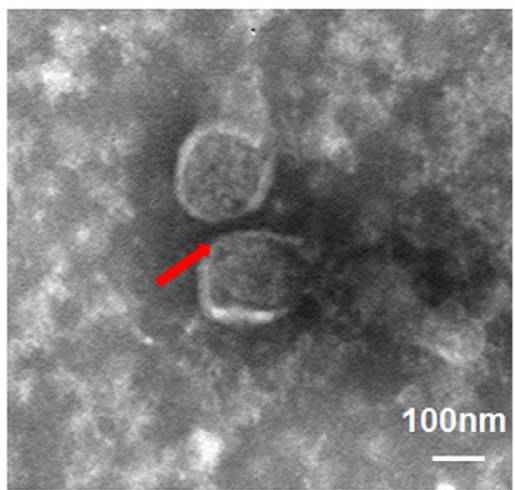

COPD

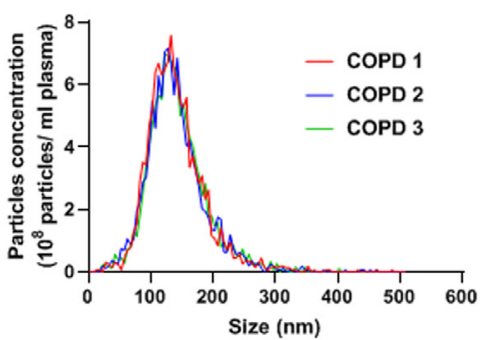

COPD

C

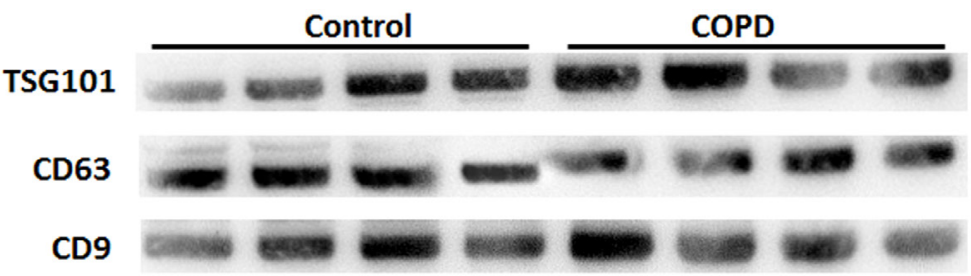

Figure 1. Isolation of plasma-derived exosomes from patients with COPD and healthy individuals. (A) Representative transmission electron microscopy images of plasma-derived exosomes isolated using the ExoQuick method. Scale bar, $100 \mathrm{~nm}$. (B) Nanoparticle tracking analysis of the plasma-derived exosomes. Representative graphs showing the concentration and size of the isolated particles are presented. $\mathrm{n}=3 / \mathrm{group}$. (C) Western blotting was performed to detect TSG101, CD63 and CD9 expression, protein markers of exosomes. TSG101, CD63 and CD9, were highly expressed in the exosomes separated from the plasma samples of patients with chronic obstructive pulmonary disease and healthy individuals. n=5/group. COPD, chronic obstructive pulmonary disease; TSG101, tumor susceptibility gene 101 .

'heterocyclic compound binding', 'nucleic acid binding' and 'transferase activity' (Fig. 3A). The primarily enriched pathways included the mTOR, chemokine, MAPK and PI3K-AKT signaling pathways (Fig. 3B).

Validation of dysregulated miRNAs in patients with COPD. The 20 exo-miRNAs with the most significant differential expression in patients with COPD were further validated via RT-qPCR. Nine of the exo-miRNAs were determined to be significantly differentially expressed by exosome sequencing, including five that were upregulated (miR-23a, miR-1, miR-574, miR-152 and miR-221) and four that were downregulated (miR-3158, miR-7706, miR-685 and miR-144) in patients with COPD compared with those in healthy individuals. These results were consistent with data obtained using RT-qPCR (Fig. 4). However, expression of the other 11 exo-miRNAs did not significantly differ between patients with COPD and healthy controls.

Construction of the exosomal miRNA-mRNA network. A network was established based on the association between the differentially expressed exo-miRNAs and mRNAs. The network presented the interaction of the five miRNAs and 98 mRNAs (Fig. 5). This network confirmed that one exo-miRNA targeted one or two mRNAs, and one mRNA was regulated by multiple miRNAs simultaneously, which suggested that a regulatory mechanism existed between exo-miRNAs and mRNAs in COPD.

Correlation between exo-miRNA expression with clinical parameters. The correlation between the exo-miRNA expression levels and the FEV1/FVC value was further assessed using linear regression. Potential confounding factors were adjusted, including age, sex, smoking status and corticosteroid therapy were adjusted using Bonferronis correction. The results indicated that the expression levels of three upregulated exo-miRNAs (miR-23a, miR-221 and miR-574) correlated significantly with the FEV1/FVC values, even after adjusting for the confounding factors. The correlation analysis between exo-miRNA and FEV1/FVC is presented in Fig. 6. miR-23a $\left(\mathrm{R}^{2}=0.706 ; \mathrm{P}<0.01 ;\right.$ Fig. 6A), miR-221 $\left(\mathrm{R}^{2}=0.757 ; \mathrm{P}<0.01\right.$; Fig. 6B) and miR-574 $\left(\mathrm{R}^{2}=0.677 ; \mathrm{P}<0.01\right.$; Fig. 6C) were analyzed.

Diagnostic value of the exo-miRNAs. ROC curves were plotted to evaluate the diagnostic value of exo-miRNAs for COPD, 
A

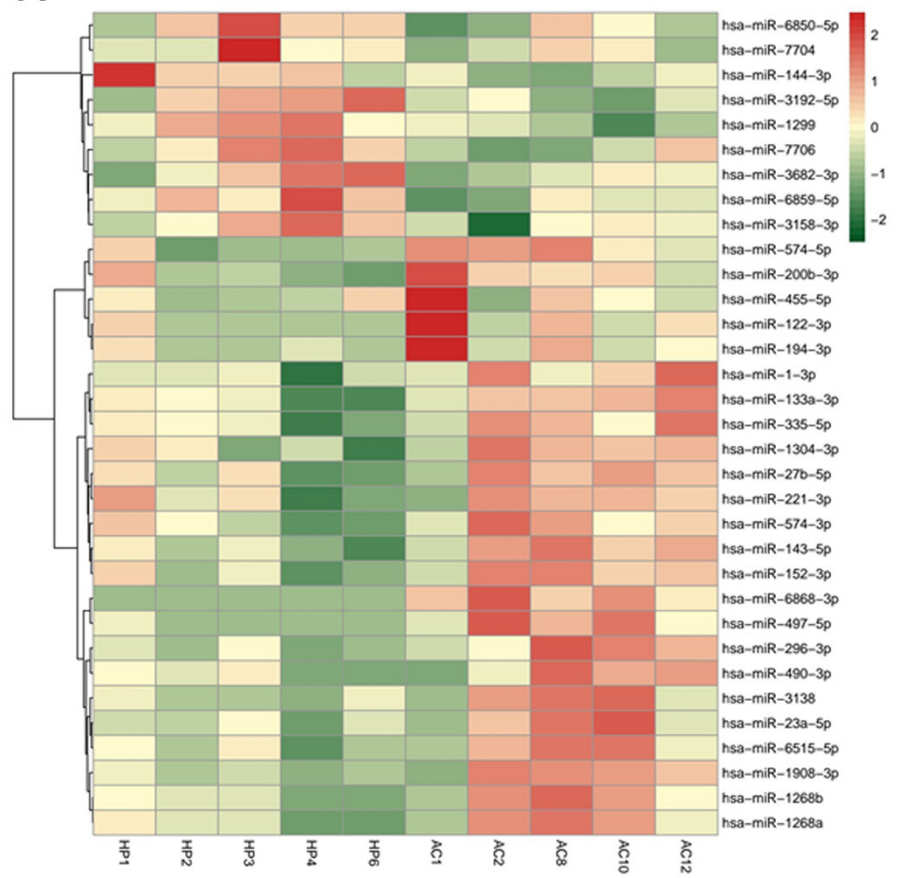

B

The Difference of miRNA Profiles

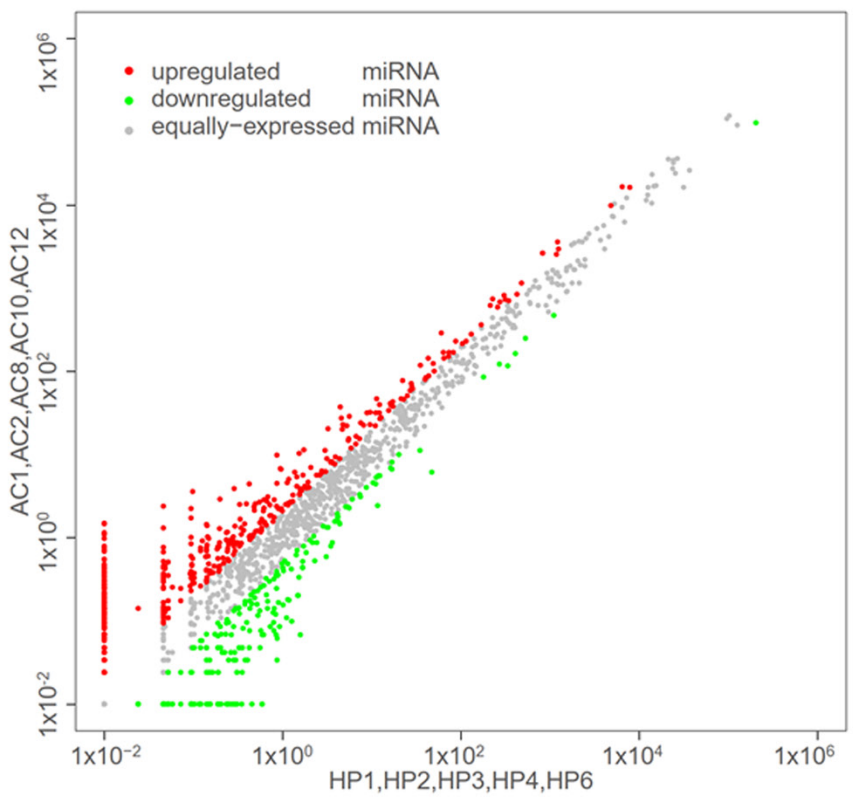

Figure 2. Hierarchical clustering (made with R language package) and scatter plot results of differentially expressed exo-miRNAs in the plasma of patients with COPD and healthy individuals. (A) Hierarchical clustering images of the exo-miRNA expression of pooled RNA samples from the plasma of patients with COPD. Brick-red indicates upregulated miRNA and green indicates downregulated miRNAs. (B) Scatter plot of miRNA expression. Values on the $\mathrm{x}$ - and $\mathrm{y}$-axis represent the normalized signal values of the samples $\left(\log _{2}\right.$ scaled). Red and green dots represent up- and downregulated miRNAs, respectively. Exo, exosomal; miRNA, microRNA; COPD, chronic obstructive pulmonary disease; HP, healthy individuals; AC, patients with COPD.

A

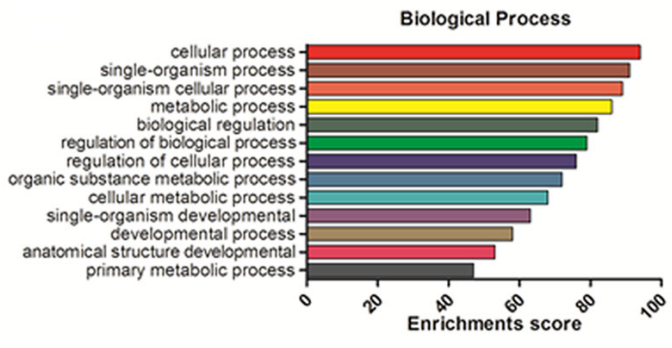

Cellular Component

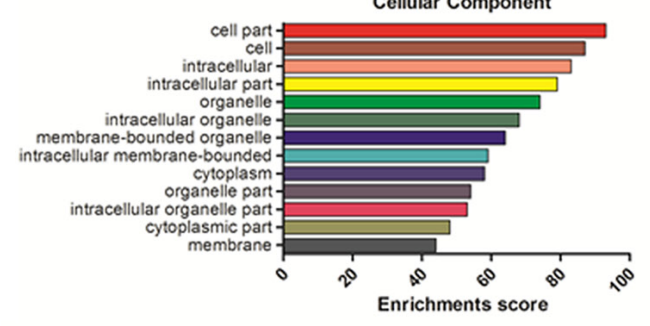

Molecular Function

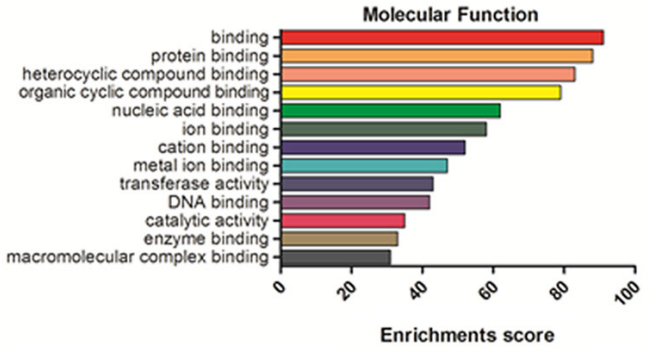

B

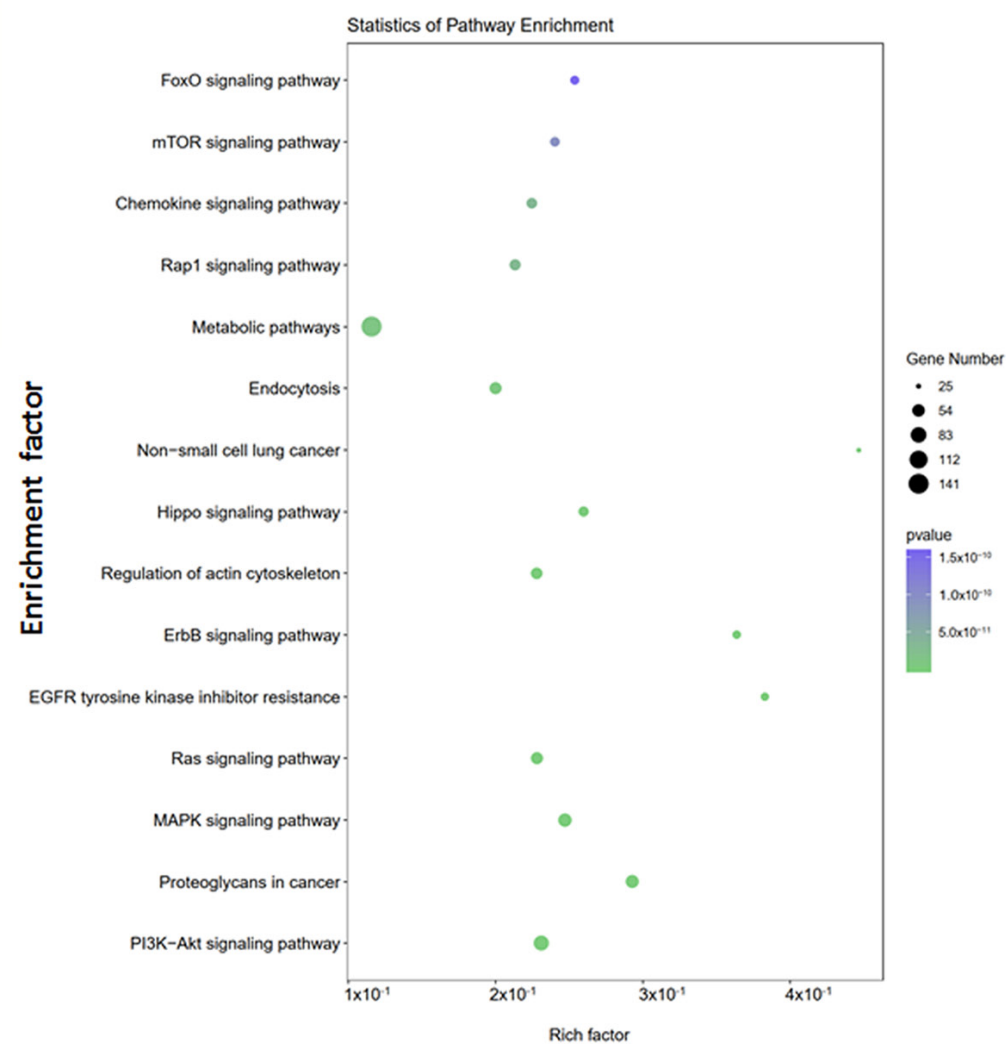

Figure 3. Functional analysis of exo-miRNAs. (A) Gene Ontology enrichment histogram of the differentially expressed exo-miRNAs. (B) Pathway analysis based on exo-miRNAs target genes. P-values represent the significance level of target gene enrichment in this pathway. Exo, exosomal; miRNAs, microRNAs. 

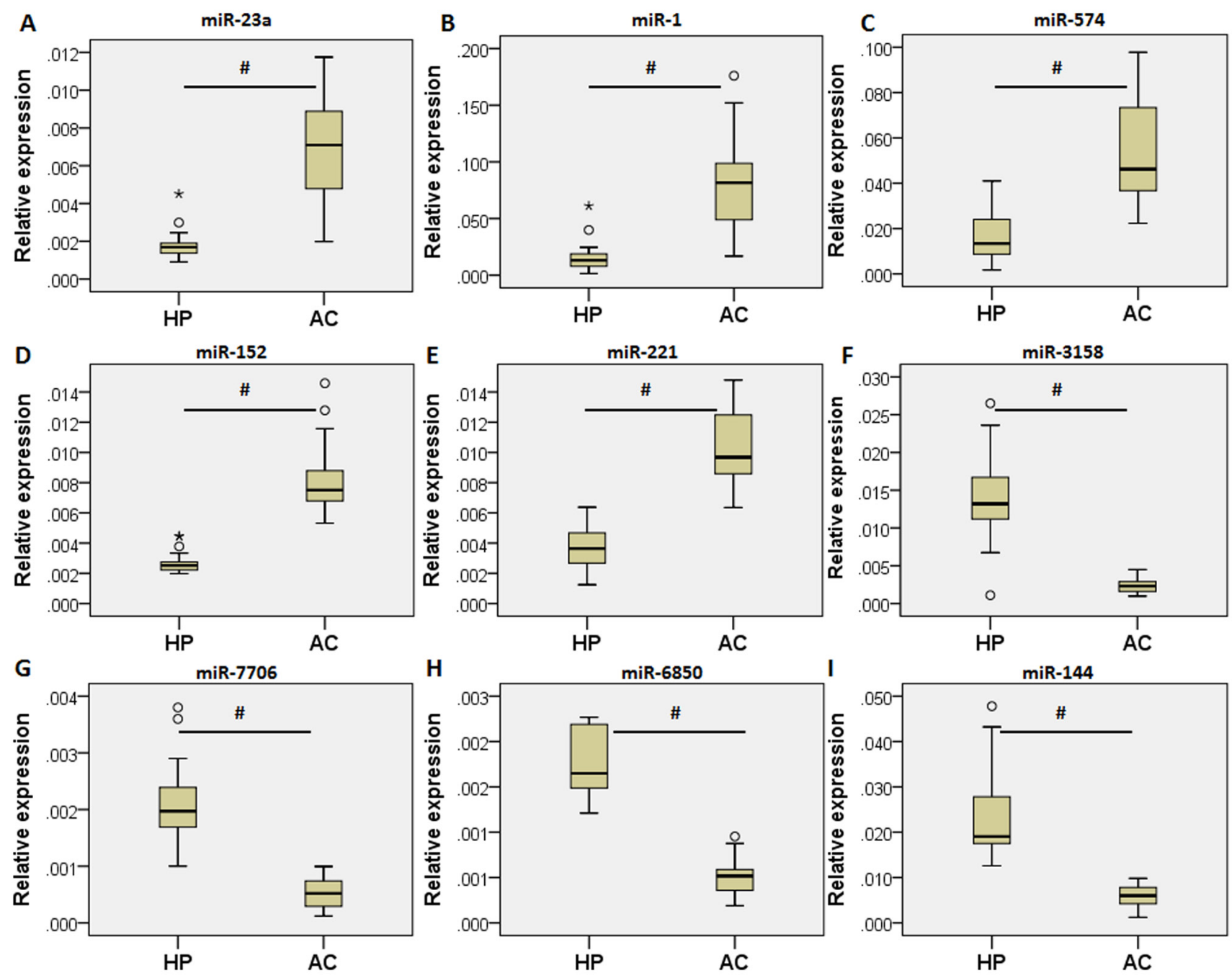

Figure 4. Validation of the differentially expressed exosomal-miRNAs (41 patients with chronic obstructive pulmonary disease and 29 healthy controls), Relative expression of (A) miR-23a, (B) miR-1, (C) miR-574, (D) miR-152, (E) miR-221, (F) miR-3158, (G) miR-7706, (H) miR-6850 and (I) miR-144 in the validation cohort. Data are presented as median. ${ }^{~} \mathrm{P}<0.05$ vs. HP. ${ }^{\circ}$ represents a mild outlier, and "represents an extreme outlier. miRNA/miR, microRNA; HP, healthy individuals; AC, patients with COPD.

which was expressed by sensitivity and specificity. The AUC of ex-miR-23a was 0.776 (95\% CI, 0.669-0.882; P<0.001). The sensitivity and specificity at the optimal cut-off were 70.3 and $61.8 \%$, respectively (Fig. 7A). The AUC of exo-miRNA-221 was 0.688 (95\% CI, 0.563-0.812; $\mathrm{P}<0.001$ ), where the sensitivity and specificity at the optimal cut-off were 69.4 and $60.2 \%$, respectively (Fig. 7B). Finally, the AUC of exo-miRNA-574 was 0.842 (95\% CI, 0.752-0.931; $\mathrm{P}<0.001)$ with a sensitivity and specificity value at the optimal cut-off of 74.3 and $65.7 \%$, respectively (Fig. 7C). Combining all three miRNAs together, the AUC increased to 0.892 (95\% CI, 0.820-0.973; $\mathrm{P}<0.001$ ). The sensitivity and specificity at the optimal cut-off were 85.7 and $77.1 \%$, respectively (Fig. 7D).

\section{Discussion}

Accumulating evidence has demonstrated that exosomes mediate a variety of molecular and cellular events by facilitating intercellular signaling $(13,15,44,45)$. Next-generation sequencing is a powerful tool that can identify novel circulating biomarkers in various diseases, such as heart disease $(46,47)$, cancer (48) and polycystic ovary syndrome (49). In recent years, the relative distribution of miRNAs in different body fluids, including blood, plasma, serum, saliva and urine have been determined (50). In addition, it was hypothesized that the components of exosomes may be altered during the development of COPD (51). Therefore, the present study performed a comprehensive analysis of exo-miRNAs that were aberrantly expressed in patients with COPD. The results suggested that three exo-miRNAs, miR-23a, miR-221 and miR-574, may serve as potential diagnostic biomarkers of COPD.

Previous studies have verified the crucial role of miRNAs in modulating gene expression under diverse conditions $(15,44)$. Dysregulation of miRNA expression in airway epithelial cells, peripheral blood and lung tissues has been associated with the pathogenesis of COPD (17,52-54). Ezzie et al (52) compared the expression of miRNAs in the lung tissue samples of COPD and smokers without COPD, which elucidated 70 miRNAs that were differentially expressed. In another study, Van Pottelberge et al (55) revealed that 34 miRNAs were differentially expressed between non-smokers and smokers without airflow restriction, where the expression of 


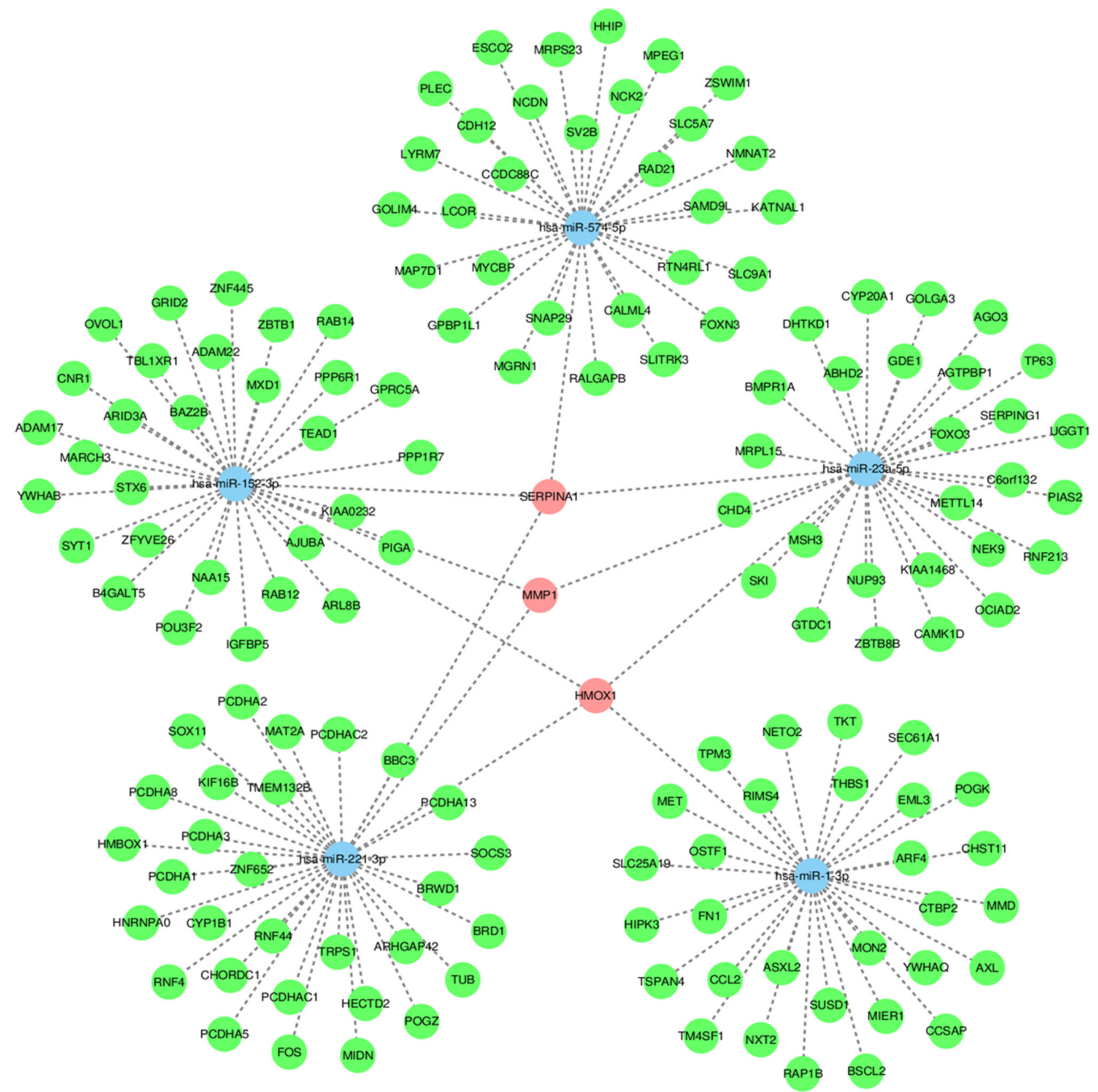

Figure 5. Regulatory network between exosomal-miRNAs and mRNAs. The miRNA-mRNA co-expression interaction network was constructed using the Cytoscape software. The network consists of five nodes (miRNA). Blue and green circles represent miRNAs and mRNAs, respectively. The pink nodes signify that the genes have a targeted regulatory relationship with two or more miRNAs. The interaction between two factors is represented by one edge. miRNA, microRNA.

eight miRNAs in smokers with COPD was significantly lower compared with that in non-smokers. The results of the present study differed from the aforementioned studies. This was mainly as miRNAs were extracted from different samples, namely airway epithelial cells, serum or lung tissues, difficulty remains in obtaining a comprehensive comparison with previous experimental results. In addition, these results may demonstrate discrepancy, arising from other factors, including different sample sizes, analytical tools and statistical methods.

Results from the current study suggested that exosomal miRNAs were differentially expressed in patients with COPD.
Furthermore, 59 differentially expressed exo-miRNAs were identified, including 39 that were upregulated and 20 that were downregulated. GO enrichment analysis provides a unified vocabulary to elaborate gene and gene product properties in various organisms (56). GO analysis in the present study indicated that the identified exo-miRNAs were enriched in various annotations associated with COPD, as demonstrated by the top enriched biological processes and molecular functions, including 'metabolic process', 'biological regulation', 'intracellular membrane-bounded organelle', 'transferase activity', 'catalytic activity' and 'enzyme binding'. 

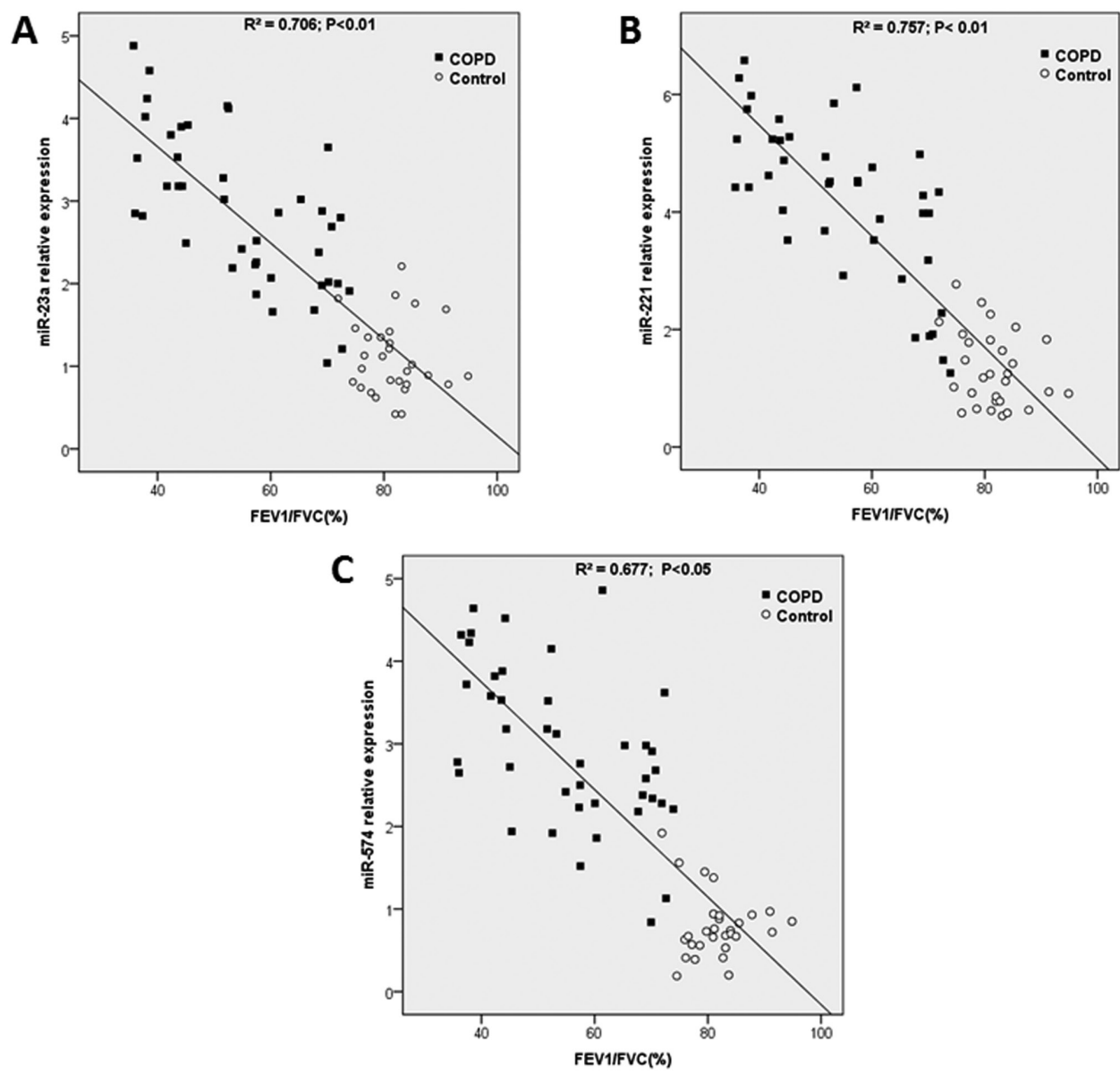

Figure 6. Correlation analysis of exo-miRNA expression and FEV1/FVC\%. Correlation between the expression of exo-miRNAs (A) miR-23a, (B) miR-221 and $(\mathrm{C})$ miR-574 independently and FEV1/FVC\% are presented as scatter plots, combined with the regression line. Data are derived from the validation cohort (n=70). Exo-miRNA, exosomal microRNA; FEV1, forced expiratory volume in the 1st second; FVC, forced vital capacity; COPD, chronic obstructive pulmonary disease.

High-throughput sequencing, such as small RNA sequencing technology makes it possible to measure the expression of almost all coding genes, which assists in identifying genes and pathways related to the development of diseases (51). In the present study, based on the KEGG pathway result, the target genes of dysregulated exo-miRNAs were involved in pathways that are closely associated with the pathophysiology of COPD, such as the mTOR, chemokine, MAPK and PI3K-Akt signaling pathways. Additionally, the present network analysis indicated a potential association between miRNAs and their target genes, suggesting that exo-miRNAs and their target genes cooperate to regulate the pathogenesis of COPD. Furthermore, to determine the function of the identified exo-miRNAs, interactions between exo-miRNAs and their target mRNAs were theoretically predicted using conserved seed-matching sequences with software for miRNA target prediction, such as TargetScan and miRDB. This network suggested the potential associations between exo-miRNAs and their target genes. The network also provided an important reference value for studying the interaction of other differentially expressed exo-miRNAs with their potential targets. The current study predicted that the interaction of exo-miRNAs and their target genes was associated with COPD. The present study found 3 common target genes [encoding $\alpha 1$-antitrypsin (SERPINA1), matrix metalloproteinase 1 (MMP1), heme oxygenase-1 (HOMX1)] of the 5 miRNAs, which are closely related to the pathogenesis of COPD. SERPINA1 has been shown to affect the susceptibility of COPD (57). Dysregulation in the production of MMP has been associated with lung matrix destruction and small airways disease in COPD (58). HOMX1 (induction attenuated senescence in chronic obstructive pulmonary disease lung fibroblasts by protecting against mitochondria dysfunction (59).

Exo-miRNAs that were differentially expressed between patients with COPD and healthy controls were screened and analyzed in an independent validation cohort. Through this method, nine differentially expressed exo-miRNAs were identified, of which five were upregulated and four were downregulated. To remove various confounding factors, linear regression analysis was performed to assess the relationship between plasma miRNA expression and FEV1/FVC. As a result, three exo-miRNAs (miR-23a, miR-221 and miR-574) were significantly correlated with FEV1/FVC after adjusting for age, sex and treatment with corticosteroids. These results suggested that these 3 exo-miRNAs may be used to assess the severity of COPD lung function.

Exosomal molecules have the potential to serve as disease biomarkers for a number of reasons. Exosomes contain specific proteins and nucleic acids that carry information 
A

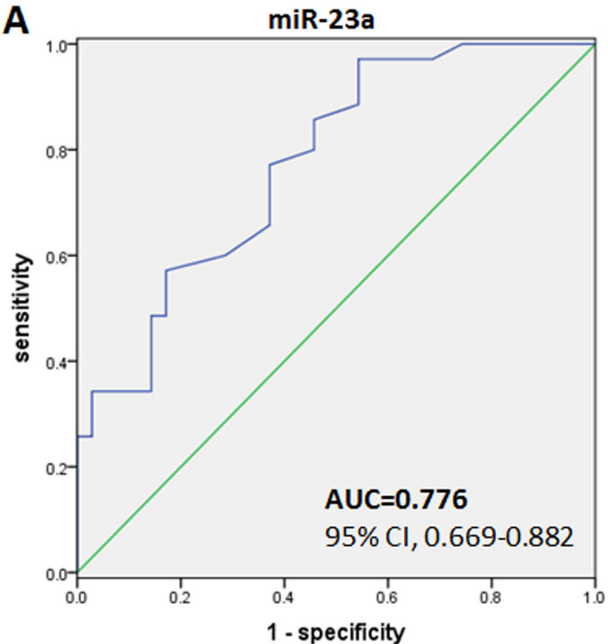

C

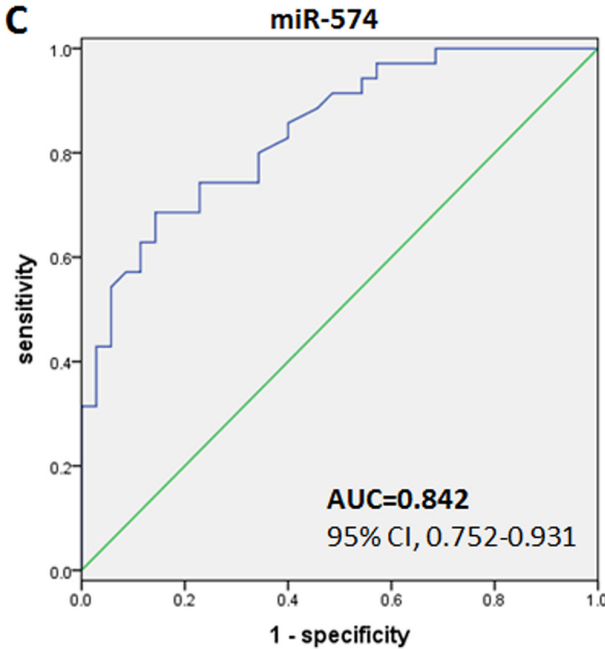

B

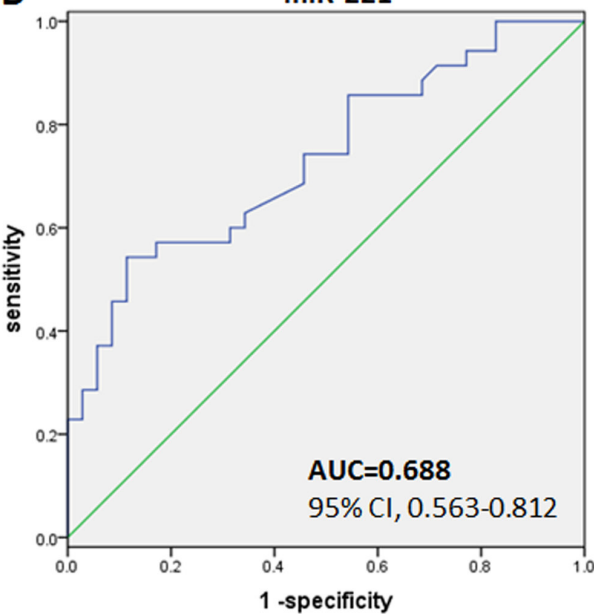

D

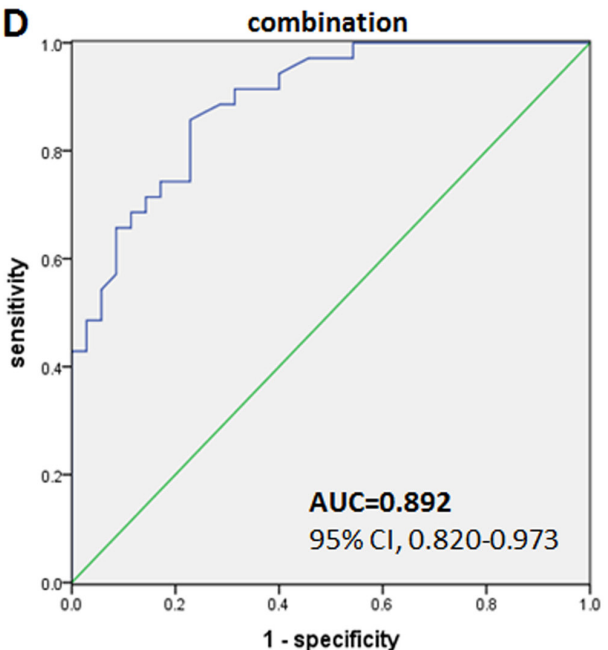

Figure 7. Receiver operating characteristic curves for three candidate exosomal-microRNAs. (A) miRNA-23a, (B) miR-221, (C) miR-574 and (D) Combination of three miRNAs. miR, microRNA; AUC, area under the curve.

regarding the physiology and microenvironment of their cells of origin (60-62). In addition, exosomes can exist in various biological fluids, including blood (25), urine (63), sputum, bronchoalveolar lavage (26), synovial fluid (64), pleural fluid and ascites (65). Due to the bilayer structure of phospholipids, exosomes are highly stable in the extracellular environment (66). Therefore, many exosomal proteins and miRNAs have been reported to be potential biomarkers of multiple diseases, particularly in cancer $(22,23)$. There is considerable evidence to support the notion that exo-miRNAs can serve an important role in multiple pulmonary diseases, such as COPD (67). ROC analysis in the present study revealed that three candidate miRNAs (miR-23a, miR-221 and miR-574) can be used as new biomarkers of COPD. Furthermore, when the three exo-miRNAs were combined together, the diagnostic efficiency was improved further.

The present study provided genome-wide profiles of exosomal miRNAs from human blood samples, demonstrating the feasibility of identifying COPD biomarkers based on exosomal miRNA profiling. Additionally, due to the double membrane structure of exosomes, exosomal miRNAs can overcome certain limitations of current biomarkers due to increased stability. However, the present study also had a number of limitations. A single center, small sample size, various forms of bias in the present study may lead to inaccurate conclusions. Therefore, a large-scale, multi-center and prospective validation study should be performed in future studies. Additionally, the underlining mechanism of the association between exosomal miRNAs and COPD remain unclear and should be investigated further.

In summary, to the best of our knowledge, the present study determined for the first time that miR-23a, miR-221 and miR-574 may serve as novel diagnostic biomarkers of COPD. The results indicated that the evaluation of exosomal miRNA expression could provide useful information regarding the diagnosis of patients with COPD.

\section{Acknowledgements}

Not applicable.

\section{Funding}

The present study was supported by grants from the National Natural Science Foundation of China (grant no. 81870039), the Jiangsu Provincial Medical Youth Talent (grant 
no. QNRC2016512) and the Taizhou Municipal Science and Technology Bureau (grant no. TS201729).

\section{Availability of data and materials}

The datasets used and/or analyzed during the current study are available in the National Center for Sequence Read Archive (SRA) data, (SRA; https://www.ncbi.nlm.nih.gov/sra/; accession no. PRJNA703816).

\section{Authors' contributions}

YS and XY designed the study and drafted the manuscript. YS and LW performed the experiments. YW and YO performed the statistical analysis. YO and HL performed sample collection. HL diagnosed the patients and revised the manuscript for important intellectual content. YS and XY confirm all the authenticity of the raw data. All authors read and approved the final manuscript.

\section{Ethics approval and consent to participate}

The present study was approved by the research ethics committee of Taizhou People's Hospital (Taizhou clinical Medical School of Nanjing Medical university; Taizhou, China). All individuals provided informed consent for the use of their samples for clinical research.

\section{Patient consent for publication}

Not applicable.

\section{Competing interests}

The authors declare that they have no competing interests.

\section{References}

1. Wang C, Xu J, Yang L, Xu Y, Zhang X, Bai C, Kang J, Ran P, Shen $\mathrm{H}$, Wen $\mathrm{F}$, et al: Prevalence and risk factors of chronic obstructive pulmonary disease in China [the China pulmonary health (CPH) study]: A national cross-sectional study. Lancet 391: 1706-1717, 2018.

2. GBD 2015 Chronic Respiratory Disease Collaborators: Global, regional, and national deaths, prevalence, disability-adjusted life years, and years lived with disability for chronic obstructive pulmonary disease and asthma, 1990-2015: A systematic analysis for the global burden of disease study 2015. Lancet Respir Med 5: 691-706, 2017.

3. Fang X, Wang X and Bai C: COPD in China: The burden and importance of proper management. Chest 139: 920-929, 2011.

4. Neumeier A and Keith R: Clinical guideline highlights for the hospitalist: The GOLD and NICE guidelines for the management of COPD. J Hosp Med 15: 240-241, 2020.

5. Rabe KF, Hurd S, Anzueto A, Barnes PJ, Buist SA, Calverley P, Fukuchi Y, Jenkins C, Rodriguez-Roisin R, van Weel C, et al: Global strategy for the diagnosis, management, and prevention of chronic obstructive pulmonary disease: GOLD executive summary. Am J Respir Crit Care Med 176: 532-555, 2007.

6. Pauwels RA and Rabe KF: Burden and clinical features of chronic obstructive pulmonary disease (COPD). Lancet 364: 613-620, 2004

7. Lopez AD and Mathers CD: Measuring the global burden of disease and epidemiological transitions: 2002-2030. Ann Trop Med Parasitol 100: 481-499, 2006.

8. Ito $\mathrm{K}$ and Barnes PJ: COPD as a disease of accelerated lung aging. Chest 135: 173-180, 2009.
9. Demedts IK, Demoor T, Bracke KR, Joos GF and Brusselle GG: Role of apoptosis in the pathogenesis of COPD and pulmonary emphysema. Respir Res 7: 53, 2006.

10. Adcock IM, Tsaprouni L, Bhavsar P and Ito K: Epigenetic regulation of airway inflammation. Curr Opin Immunol 19: 694-700, 2007.

11. Wan ES and Silverman EK: Genetics of COPD and emphysema. Chest 136: 859-866, 2009.

12. Bartel DP: MicroRNAs: Genomics, biogenesis, mechanism, and function. Cell 116: 281-297, 2004.

13. Hough KP, Chanda D, Duncan SR, Thannickal VJ and Deshane JS: Exosomes in immunoregulation of chronic lung diseases. Allergy 72: 534-544, 2017.

14. Murgoci AN, Duhamel M, Raffo-Romero A, Mallah K, Aboulouard S, Lefebvre C, Kobeissy F, Fournier I, Zilkova M, Maderova D, et al: Location of neonatal microglia drives small extracellular vesicles content and biological functions in vitro. J Extracell Vesicles 9: 1727637, 2020.

15. Nana-Sinkam SP, Acunzo M, Croce $C M$ and Wang $K$ : Extracellular vesicle biology in the pathogenesis of lung disease. Am J Respir Crit Care Med 196: 1510-1518, 2017.

16. Stolzenburg LR and Harris A: The role of microRNAs in chronic respiratory disease: Recent insights. Biol Chem 399: 219-234, 2018.

17. De Smet EG, Mestdagh P, Vandesompele J, Brusselle GG and Bracke KR: Non-coding RNAs in the pathogenesis of COPD. Thorax 70: 782-791, 2015 .

18. Molina-Pinelo S, Pastor MD, Suarez R, Romero-Romero B, González De la Peña M, Salinas A, García-Carbonero R, De Miguel MJ, Rodríguez-Panadero F, Carnero A and Paz-Ares L: MicroRNA clusters: Dysregulation in lung adenocarcinoma and COPD. Eur Respir J 43: 1740-1749, 2014.

19. Robbins PD and Morelli AE: Regulation of immune responses by extracellular vesicles. Nat Rev Immunol 14: 195-208, 2014.

20. Colombo M, Raposo G and Théry C: Biogenesis, secretion, and intercellular interactions of exosomes and other extracellular vesicles. Annu Rev Cell Dev Biol 30: 255-289, 2014.

21. Yoshioka Y, Konishi Y, Kosaka N, Katsuda T, Kato T and Ochiya T: Comparative marker analysis of extracellular vesicles in different human cancer types. J Extracell Vesicles 2, 2013.

22. Mirzaei H, Sahebkar A, Jaafari MR, Goodarzi M and Mirzaei HR: Diagnostic and therapeutic potential of exosomes in cancer: The beginning of a new tale? J Cell Physiol 232: 3251-3260, 2017.

23. Saadatpour L, Fadaee E, Fadaei S, Nassiri Mansour R, Mohammadi M, Mousavi SM, Goodarzi M, Verdi J and Mirzaei H: Glioblastoma: Exosome and microRNA as novel diagnosis biomarkers. Cancer Gene Ther 23: 415-418, 2016.

24. Sun L, Zhu W, Zhao P, Wang Q, Fan B, Zhu Y, Lu Y, Chen Q, Zhang $\mathrm{J}$ and Zhang $\mathrm{F}$ : Long noncoding RNA UCA1 from hypoxia-conditioned hMSC-derived exosomes: A novel molecular target for cardioprotection through miR-873-5p/XIAP axis. Cell Death Dis 11: 696, 2020.

25. Caby MP, Lankar D, Vincendeau-Scherrer C, Raposo G and Bonnerot C: Exosomal-like vesicles are present in human blood plasma. Int Immunol 17: 879-887, 2005.

26. Admyre C, Grunewald J, Thyberg J, Gripenbäck S, Tornling G, Eklund A, Scheynius A and Gabrielsson S: Exosomes with major histocompatibility complex class II and co-stimulatory molecules are present in human BAL fluid. Eur Respir J 22: 578-583, 2003.

27. Porro C, Lepore S, Trotta T, Castellani S, Ratclif L, Battaglino A, Di Gioia S, Martínez MC, Conese M and Maffione AB: Isolation and characterization of microparticles in sputum from cystic fibrosis patients. Respir Res 11: 94, 2010.

28. Salimian J, Mirzaei H, Moridikia A, Harchegani AB, Sahebkar A and Salehi H: Chronic obstructive pulmonary disease: MicroRNAs and exosomes as new diagnostic and therapeutic biomarkers. J Res Med Sci 23: 27, 2018.

29. Lener T, Gimona M, Aigner L, Börger V, Buzas E, Camussi G, Chaput N, Chatterjee D, Court FA, Del Portillo HA, et al: Applying extracellular vesicles based therapeutics in clinical trials-an ISEV position paper. J Extracell Vesicles 4: 30087, 2015.

30. Dang X, Qu X, Wang W, Liao C, Li Y, Zhang X, Xu D, Baglole CJ, Shang D and Chang Y: Bioinformatic analysis of microRNA and mRNA regulation in peripheral blood mononuclear cells of patients with chronic obstructive pulmonary disease. Respir Res 18: 4, 2017.

31. Kara M, Kirkil G and Kalemci S: Differential expression of MicroRNAs in chronic obstructive pulmonary disease. Adv Clin Exp Med 25: 21-26, 2016. 
32. Wang L and Zhang L: Circulating exosomal miRNA as diagnostic biomarkers of neurodegenerative diseases. Front Mol Neurosci 13: 53, 2020.

33. Albitar HAH and Iyer VN: Adherence to global initiative for chronic obstructive lung disease guidelines in the real world: Current understanding, barriers, and solutions. Curr Opin Pulm Med 26: 149-154, 2020

34. Sundar IK, Li D and Rahman I: Small RNA-sequence analysis of plasma-derived extracellular vesicle miRNAs in smokers and patients with chronic obstructive pulmonary disease as circulating biomarkers. J Extracell Vesicles 8: 1684816, 2019.

35. Théry C, Amigorena S, Raposo G and Clayton A: Isolation and characterization of exosomes from cell culture supernatants and biological fluids. Curr Protoc Cell Biol Chapter 3: Unit 322 2006.

36. Mehdiani A, Maier A, Pinto A, Barth M, Akhyari P and Lichtenberg A: An innovative method for exosome quantification and size measurement. J Vis Exp: 50974, 2015.

37. Mortazavi A, Williams BA, McCue K, Schaeffer L and Wold B Mapping and quantifying mammalian transcriptomes by RNA-Seq. Nat Methods 5: 621-628, 2008.

38. Robinson MD, McCarthy DJ and Smyth GK: edgeR: A bioconductor package for differential expression analysis of digital gene expression data. Bioinformatics 26: 139-140, 2010.

39. Parker VL, Cushen BF, Gavriil E, Marshall B, Waite S, Pacey A and Heath PR: Comparison and optimisation of microRNA extraction from the plasma of healthy pregnant women. Mol Med Rep 23: 1, 2021

40. Livak KJ and Schmittgen TD: Analysis of relative gene expression data using real-time quantitative PCR and the 2(-Delta Delta C(T)) method. Methods 25: 402-408, 2001.

41. Xie C, Mao X, Huang J, Ding Y, Wu J, Dong S, Kong L, Gao G Li CY and Wei L: KOBAS 2.0: A web server for annotation and identification of enriched pathways and diseases. Nucleic Acids Res 39 (Web Server Issue): W316-W322, 2011.

42. Shannon P, Markiel A, Ozier O, Baliga NS, Wang JT, Ramage D, Amin N, Schwikowski B and Ideker T: Cytoscape: A software environment for integrated models of biomolecular interaction networks. Genome Res 13: 2498-2504, 2003.

43. Barabási AL and Oltvai ZN: Network biology: Understanding the cell's functional organization. Nat Rev Genet 5: 101-113, 2004.

44. Yáñez-MóM, Siljander PR, Andreu Z, Zavec AB, Borràs FE, Buzas EI, Buzas K, Casal E, Cappello F, Carvalho J, et al: Biological properties of extracellular vesicles and their physiological functions. J Extracell Vesicles 4: 27066, 2015.

45. Fujita Y, Kosaka N, Araya J, Kuwano K and Ochiya T: Extracellular vesicles in lung microenvironment and pathogenesis. Trends Mol Med 21: 533-542, 2015.

46. Wang C, Song C, Liu Q, Zhang R, Fu R, Wang H, Yin D, Song W, Zhang $\mathrm{H}$ and Dou K: Gene expression analysis suggests immunological changes of peripheral blood monocytes in the progression of patients with coronary artery disease. Front Genet 12: 641117, 2021.

47. Hou Z, Qin X, Hu Y, Zhang X, Li G, Wu J, Li J, Sha J, Chen J, $\mathrm{Xia} \mathrm{J}$, et al: Longterm exercise-derived exosomal miR-342-5p: A novel exerkine for cardioprotection. Circ Res 124: 1386-1400, 2019.

48. Han Y, Chen J, Zhao X, Liang C, Wang Y, Sun L, Jiang Z, Zhang Z, Yang R, Chen J, et al: MicroRNA expression signatures of bladder cancer revealed by deep sequencing. PLoS One 6: e18286, 2011.

49. Wang LP, Peng XY, Lv XQ, Liu L, Li XL, He X, Lv F, Pan Y, Wang L, Liu KF and Zhang XM: High throughput circRNAs sequencing profile of follicle fluid exosomes of polycystic ovary syndrome patients. J Cell Physiol, Feb 18, 2019 (Epub ahead of print). doi: https://doi.org/10.1002/jcp.28201.

50. El-Mogy M, Lam B, Haj-Ahmad TA, McGowan S, Yu D, Nosal L, Rghei N, Roberts P and Haj-Ahmad Y: Diversity and signature of small RNA in different bodily fluids using next generation sequencing. BMC Genomics 19: 408, 2018.

51. 't Hoen PA, Ariyurek Y, Thygesen HH, Vreugdenhil E, Vossen RH, de Menezes RX, Boer JM, van Ommen GJ and den Dunnen JT: Deep sequencing-based expression analysis shows major advances in robustness, resolution and inter-lab portability over five microarray platforms. Nucleic Acids Res 36: e141, 2008.
52. Ezzie ME, Crawford M, Cho JH, Orellana R, Zhang S, Gelinas R, Batte K, Yu L, Nuovo G, Galas D, et al: Gene expression networks in COPD: microRNA and mRNA regulation. Thorax 67: 122-131, 2012.

53. Akbas F, Coskunpinar E, Aynaci E, Oltulu YM and Yildiz P Analysis of serum micro-RNAs as potential biomarker in chronic obstructive pulmonary disease. Exp Lung Res 38: 286-294, 2012.

54. Schembri F, Sridhar S, Perdomo C, Gustafson AM, Zhang X, Ergun A, Lu J, Liu G, Zhang X, Bowers J, et al: MicroRNAs as modulators of smoking-induced gene expression changes in human airway epithelium. Proc Natl Acad Sci USA 106: 2319-2324, 2009.

55. Van Pottelberge GR, Mestdagh P, Bracke KR, Thas O, van Durme YM, Joos GF, Vandesompele J and Brusselle GG: MicroRNA expression in induced sputum of smokers and patients with chronic obstructive pulmonary disease. Am J Respir Crit Care Med 183: 898-906, 2011.

56. Ashburner M, Ball CA, Blake JA, Botstein D, Butler H, Cherry JM, Davis AP, Dolinski K, Dwight SS, Eppig JT, et al: Gene ontology: Tool for the unification of biology. The gene ontology consortium. Nat Genet 25: 25-29, 2000.

57. Nakamura H: Genetics of COPD. Allergol Int 60: 253-258, 2011.

58. Ostridge K, Williams N, Kim V, Bennett M, Harden S, Welch L, Bourne S, Coombs NA, Elkington PT, Staples KJ and Wilkinson TM: Relationship between pulmonary matrix metalloproteinases and quantitative CT markers of small airways disease and emphysema in COPD. Thorax 71: 126-132, 2016.

59. Even B, Fayad-Kobeissi S, Gagliolo JM, Motterlini R, Boczkowski J, Foresti R and Dagouassat M: Heme oxygenase-1 induction attenuates senescence in chronic obstructive pulmonary disease lung fibroblasts by protecting against mitochondria dysfunction. Aging Cell 17: e12837, 2018.

60. de Jong OG, Verhaar MC, Chen Y, Vader P, Gremmels H, Posthuma G, Schiffelers RM, Gucek M and van Balkom BW: Cellular stress conditions are reflected in the protein and RNA content of endothelial cell-derived exosomes. J Extracell Vesicles 1, 2012.

61. Beninson LA and Fleshner M: Exosomes: An emerging factor in stress-induced immunomodulation. Semin Immunol 26: 394-401, 2014

62. Iraci N, Leonardi T, Gessler F, Vega B and Pluchino S: Focus on extracellular vesicles: Physiological role and signalling properties of extracellular membrane vesicles. Int J Mol Sci 17: 171, 2016.

63. Pisitkun T, Shen RF and Knepper MA: Identification and proteomic profiling of exosomes in human urine. Proc Natl Acad Sci USA 101: 13368-13373, 2004.

64. Song JE, Kim JS, Shin JH, Moon KW, Park JK, Park KS and Lee EY: Role of synovial exosomes in osteoclast differentiation in inflammatory arthritis. Cells 10: 120, 2021.

65. Hu Y, Qi C, Liu X, Zhang C, Gao J, Wu Y, Yang J, Zhao Q, Li J, Wang X and Shen L: Malignant ascites-derived exosomes promote peritoneal tumor cell dissemination and reveal a distinct miRNA signature in advanced gastric cancer. Cancer Lett 457: 142-150, 2019

66. Sun L, Zhu W, Zhao P, Zhang J, Lu Y, Zhu Y, Zhao W, Liu Y, Chen Q and Zhang F: Down-regulated exosomal MicroRNA-221-3p derived from senescent mesenchymal stem cells impairs heart repair. Front Cell Dev Biol 8: 263, 2020.

67. Chen J, Hu C and Pan P: Extracellular vesicle MicroRNA transfer in lung diseases. Front Physiol 8: 1028, 2017.

This work is licensed under a Creative Commons Attribution-NonCommercial-NoDerivatives 4.0 International (CC BY-NC-ND 4.0) License. 